\title{
Ad Hoc Reviewers 1994
}

Aaslid, R. Adams, H. Aichner, F. Amarenco, P. Assmann, G. Barnford, J. Baron, J.-C. Bousser, M.-G. Boysen, G. Brandt T. Brott, T. Upiner, U.R. Caplan, L. Carolei, A. Chambers, B. Cummings, J. Daffertshofer, M. de Merloose, P. de Reuck, J. de Tribolet, N. Di Pasquale, G. Eicke, M. Elger, Ch. Erkinjuntti, T. Fassbender, K. Fazekas, F. Fieschi, C. Fisher, M. Fisher-Miller, C. Frackowiak, R.SJ. Frahm, J. Fnrlan, A. Gautier, J.C. Ginsberg, $\mathrm{M}$. 
Hachinski, V. Hacke, W. Hankey, G. Hart, R. C|f ss, W.D. Homberg, V. Hossmann, K.-A. Hupperts, A. Inzitari, D. Johansson, B. Kappenberger, L. Kase, C. Kienast, J. Klingelhofer, J. Kuschinsky, W. Lenzi, G.L. Levi, F. Mattle, H. Mauritz, K.-H. Meienberg, O. Mohr, J.P. Moulin, T. Miiller, H. Niederkorn, K. Nighoghossian, N. Norris, J. Norrving, B. Orgogozo, J.-M. Pessin, M. Pohlmann-Eden, B. frockop, L. Rautenberg, W. Ricci, S. Ringelstein, E.B. 
Russell, D. Sarasin, F. Schafer, H. Scharf, R.E.

Schmid-Schonbein, G.W. Schmid-Schonbein, H. Schmiedek, P. Schwartz, A. Steinke, W. Tegeler, C. Tietjen, G. Toole, J.F. van de Loo, J. van Gijn, J. Vingerhoets, F. von Cramon, D.Y. Waeber, B. Wahlgren, N.G. Warach, S. Warlow, C.P. Waxman, G.S. Whisnant, J.P. Widder, B. Woimant, F. Yamaguchi, T. Zeumer, H. 